\title{
DIA-based proteome profiling of nasopharyngeal swabs from COVID-19 patients
}

Dong-Gi Mun ${ }^{1}$, Patrick M. Vanderboom ${ }^{1}$, Anil K. Madugundu ${ }^{1,2,3,4}$, Kishore Garapati ${ }^{1,3,4}$, Chavan Sandip ${ }^{1}$, Jane A. Peterson ${ }^{5}$, Mayank Saraswat ${ }^{1,3,4}$ and Akhilesh Pandey ${ }^{1,2,6^{*}}$

${ }^{1}$ Department of Laboratory Medicine and Pathology, Mayo Clinic, Rochester, MN 55905, USA

${ }^{2}$ Center for Molecular Medicine, National Institute of Mental Health and Neurosciences, Hosur Road, Bangalore, 560029, Karnataka, India

${ }^{3}$ Institute of Bioinformatics, International Technology Park, Bangalore, 560066, Karnataka, India ${ }^{4}$ Manipal Academy of Higher Education, Manipal, 576104, Karnataka, India ${ }^{5}$ Proteomics Core, Medical Genome Facility, Mayo Clinic, Rochester, MN 55905, USA

${ }^{6}$ Center for Individualized Medicine, Mayo Clinic, Rochester, MN 55905, USA

*Correspondence should be addressed to Akhilesh Pandey (pandey.akhilesh@mayo.edu). Akhilesh Pandey, M.D., Ph.D.

Professor

Department of Laboratory Medicine and Pathology, Mayo Clinic 200 First ST SW, Rochester, MN 55905

Phone: 507-293-9564

Email: pandey.akhilesh@mayo.edu

Figure S1. Distribution of protein identified in multiple samples and enriched canonical pathways S2

Figure S2. A representative example of detected viral peptide AYNVTQAFGR

Figure S3. The top scoring networks in IPA

Table S1. Mass spectrometry settings of diaPASEF experiment.

Table S2. The spectral library used for interpretation of diaPASEF data.

Table S3. The list of proteins quantified from 90 diaPASEF experiments. 
A

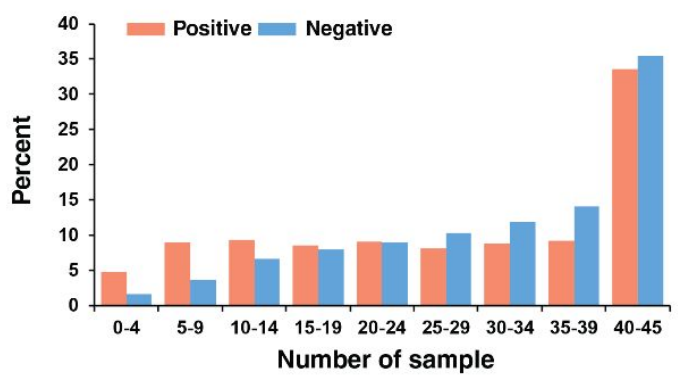

B

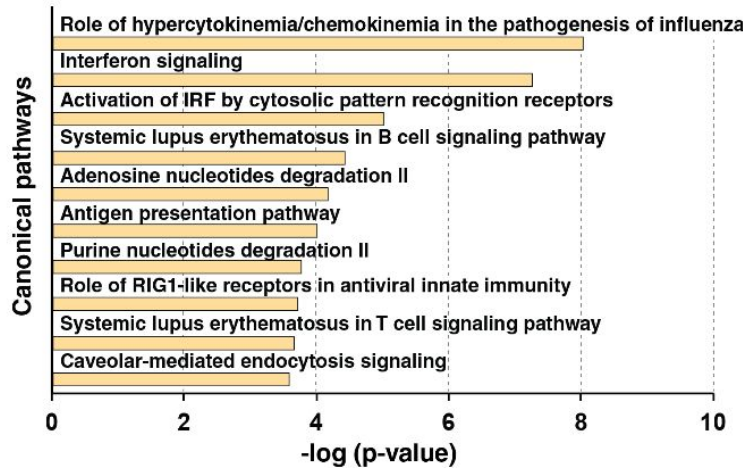

Figure S1. (A) Distribution of proteins identified from multiple samples. (B) Enriched canonical pathways in IPA. 
A

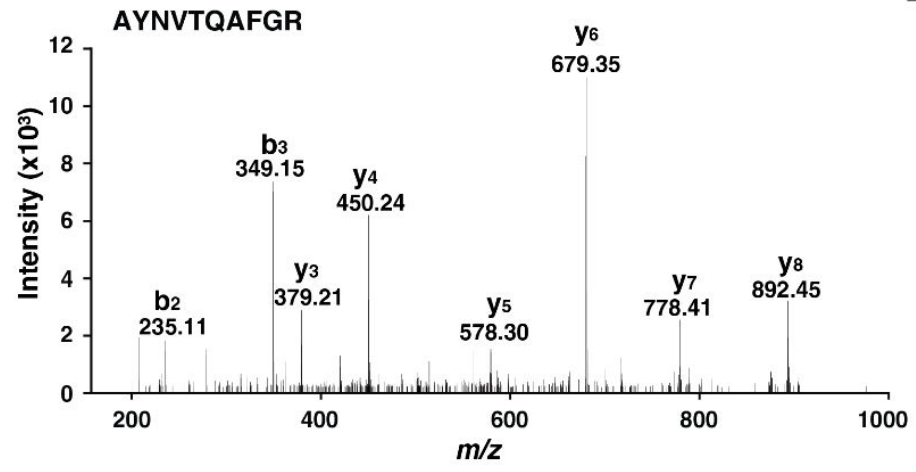

B

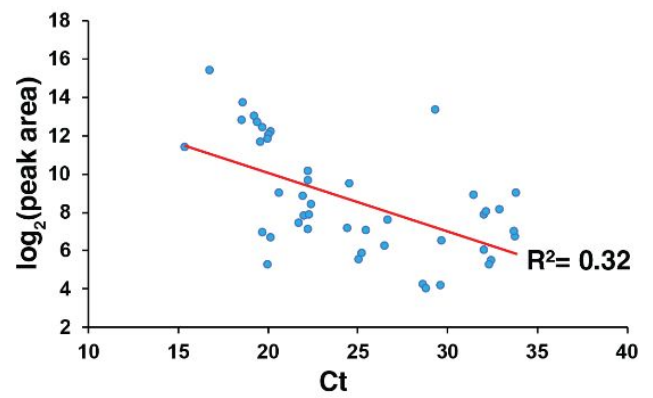

Figure S2. (A) A representative MS/MS spectrum of viral peptide AYNVTQAFGR. (B) Linear regression of RT-PCR Ct values and peak area of the detected peptide AYNVTQAFGR. 

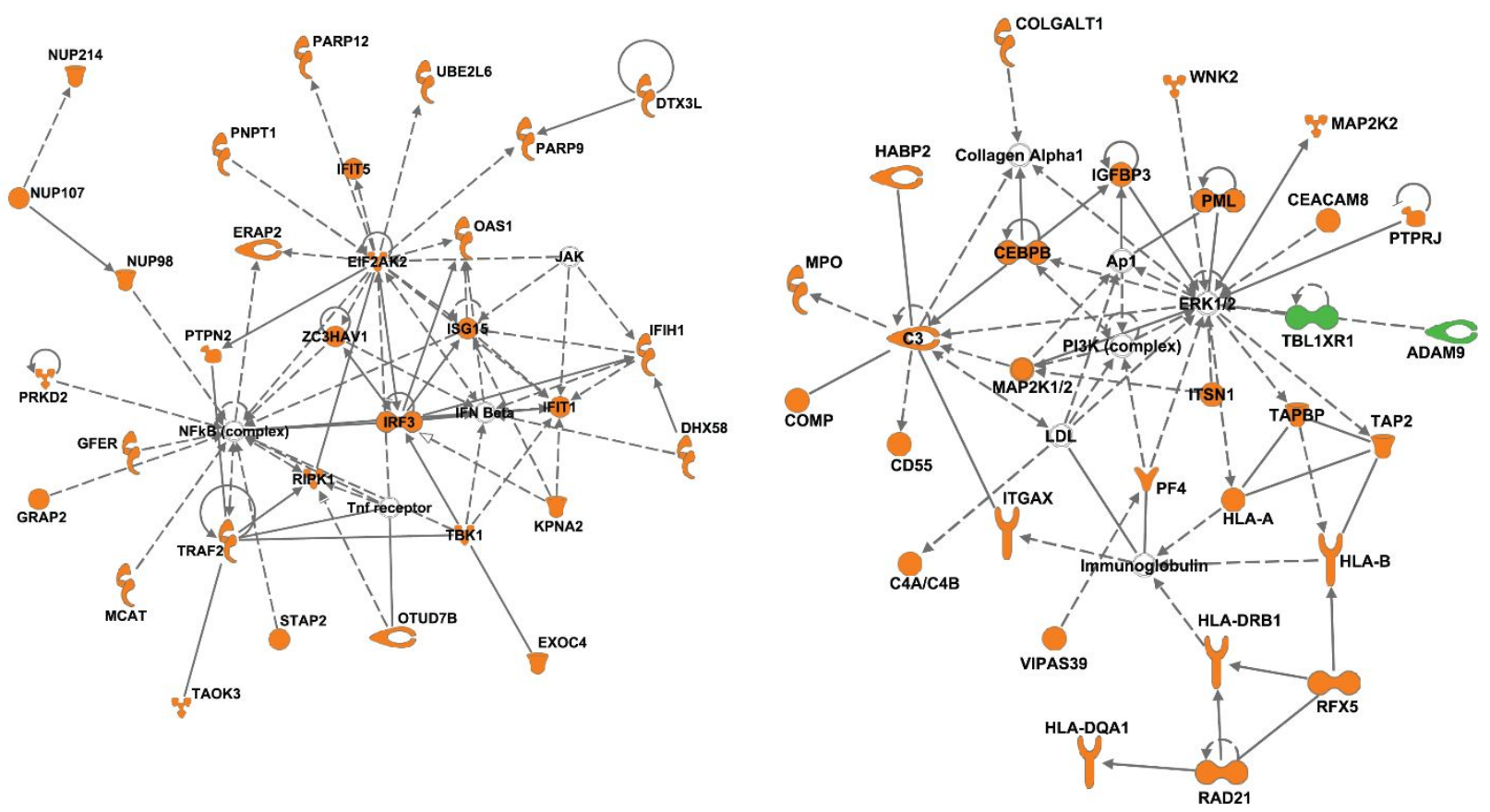

Figure S3. (A) The top scoring networks in IPA under inflammatory response. (B) The network assigned under the category of immunological/hematological/dermatological diseases. Nodes in orange and green color represent upregulated and downregulated protein, respectively. 